\title{
Sequential processes in the generalization and transfer of stimulus control
}

\author{
JAMES V. COUCH, JEREMIAH P. COLLINS, and JOHN W. DONAHOE \\ University of Massachusetts, Amherst, Massachusetts
}

\begin{abstract}
Prior research indicated that a training sequence consisting of a negative stimulus followed by a positive stimulus constitutes the minimal condition for the production of postdiscrimination phenomena typically observed after training with random sequences of the discriminanda. The present experiments, employing multiple schedules with pigeon subjects, confirmed the earlier findings but indicated that they are restricted to procedures in which the reinforcing stimulus may acquire a discriminative function that competes with the control exerted by the nominal discriminanda. The sequences in which the discriminanda were presented did not differentially affect subsequent measures of generalization and transfer if the discriminative function of reinforcement were degraded either by introducing some reinforcers during the negative stimulus (Experiment 1) or by omitting some reinforcers during the positive stimulus (Experiment 2). It was concluded that the sequence in which the discriminanda are presented during discrimination training does not contribute fundamentally to the processes responsible for discrimination formation with random training sequences.
\end{abstract}

The purpose of these experiments was to assess the generality of previous findings regarding the effects of the sequence of presentation of discriminanda on subsequent measures of stimulus control.

In the major reference experiment (Ellis, 1970), postdiscrimination performance after exposure to one of several nonrandom sequences of discriminanda was compared with that after exposure to the more usual randomly alternating sequence. One nonrandom sequence consisted of multiple training sessions, each of which was composed of massed presentations of the positive stimulus followed by massed presentations of the negative stimulus. The daily sequence of acquisition-using a variableinterval (VI) reinforcement schedule-followed by extinction was designated the $\mathrm{AE}$ sequence. A second nonrandom sequence was composed of an otherwise comparable exposure to massed extinction followed by massed acquisition, and was termed the EA sequence. The effects of the nonrandom sequences on stimulus control were evaluated by two measures: the postdiscrimination gradient of stimulus generalization and transfer to $a$ random $(R)$ sequence of discriminanda. By both measures, stimulus control following training with the $\mathrm{AE}$ sequence differed

These experiments were supported by NIMH Research Grant MH-17395 and a Biomedical Research Support Grant to the University of Massachusetts-Amherst. James V. Couch is now at the Department of Psychology, James Madison University, Harrisonburg, Virginia 22802; Jeremiah P. Collins is now at the Department of Psychiatry, Medical School, University of California at Los Angeles, Los Angeles, California 90024. Requests for reprints should be addressed to John W. Donahoe, Department of Psychology, University of Massachusetts-Amherst, Amherst, Massachusetts 01003. from the typical postdiscrimination performance found with the EA and R sequences. The generalization gradients following the $\mathrm{AE}$ sequence more closely resembled those found after nondifferential training in that the gradients were not elevated in the region opposite to the negative stimulus (Hanson, 1959). Furthermore, transfer from the $A E$ to the $R$ sequence was poor in that differential responding during the discriminanda did not occur without additional training. (For related work using a single session of exposure to the $\mathrm{AE}$ sequence, see: Ellis, 1970; Honig, Thomas, \& Guttman, 1959; and Rosen \& Terrace, 1975.) With the EA sequence, however, performance was indistinguishable from that after the $\mathbf{R}$ sequence. Postdiscrimination gradients were elevated in the region opposite to the negative stimulus (the peak shift effect), and transfer to the $\mathrm{R}$ sequence was strong and immediate.

The differential effects of $\mathrm{AE}$ and $\mathrm{EA}$ sequences on postdiscrimination performance have been interpreted to mean that the EA sequence is the origin of stimulus control in standard training procedures employing $R$ sequences; that is, the EA sequence is both necessary and sufficient for stimulus control (Ellis, 1970). For this reason, a number of investigators have suggested that nonrandom sequences of discriminanda may provide useful tools for identifying the minimal conditions essential for various discrimination phenomena (Rosen \& Terrace, 1975) and for investigating the processes involved in discrimination formation (Hinson \& Malone, 1980).

There is empirical precedence for supposing that sequential effects contribute importantly to discrimination performance. With discrimination training using $\mathbf{R}$ sequences, the rate of responding during a 
stimulus is increased when the previous stimulus controls a low rate and is decreased when the previous stimulus controls a high rate. This inverse relationship between the rates of responding during present and previous stimuli is termed local contrast (Malone \& Staddon, 1973; Nevin \& Shettleworth, 1966). Local contrast is, in turn, linked to another characteristic of discrimination performance: contrast shoulders, or edge effects (Hinson \& Malone, 1980). Edge effects take the form of an exaggerated difference in the rate of responding during stimuli near the boundary between different reinforcement schedules (Blough, 1975; Essock \& Blough, 1977). Finally, the sequence of stimuli presented during generalization testing is known to affect the shape of the postdiscrimination gradient. An $R$ sequence during generalization testing steepens the gradient on the side nearest the negative stimulus and elevates the gradient on the side opposite to the negative stimulus compared with gradients based upon exposure to only one stimulus within each test session (Donahoe, McCroskery, \& Richardson, 1970).

Before using nonrandom training sequences to study stimulus control, however, it is prudent to evaluate the generality of the differential effects of $\mathrm{AE}$ and $\mathrm{EA}$ sequences on postdiscrimination performance. The effects of nonrandom sequences have thus far been examined only with the stimulus dimension of visual intensity and with simple schedules involving the presence vs. the absence of reinforcement during the two discriminanda (Ellis, 1970). In Experiment 1 of this report, the work with nonrandom sequences was systematically replicated using the more commonly employed dimension of visual wavelength instead of intensity. A second major purpose of Experiment 1 was to examine differential sequence effects using schedules in which reinforcement occurred during both discriminanda. The persistence of sequence effects with this latter manipulation is crucial because of a potentially serious confound present in previous work. When the nominal discriminanda in nonrandom sequences are differentially associated with the presence vs. the absence of reinforcement, the reinforcing stimulus itself may acquire a discriminative function. With random sequences of discriminanda or with nonrandom sequences in which reinforcers may occur during both discriminanda, a discriminative function of the reinforcer is precluded because present reinforcers are not predictive of reinforcers for subsequent responses. The possible discriminative function of reinforcement was pursued further in Experiment 2, which employed the original intensity dimension used by Ellis (1970) and a different procedural manipulation to explore the potential confound between the nominal discriminanda and the reinforcing stimulus.

\section{EXPERIMENT 1}

As in the Ellis (1970) experiment, three sequences of stimuli were used during discrimination training: two nonrandom (AE and EA) sequences and a random ( $R$ ) sequence. The discriminative stimulus during A periods was correlated with a variableinterval 1-min (VI 1) schedule of reinforcement, and the stimulus during E periods was correlated with an extinction schedule (EXT).

Experiment 1 also examined the possibility that nonrandom sequences permitted the reinforcer to acquire a discriminative function that interfered with the stimulus control exerted by the nominal discriminative stimulus dimension, in this case wavelength. With $\mathrm{AE}$ and $\mathrm{EA}$ sequences, both the reinforcement and the nominal discriminative stimulus are predictive of subsequent reinforcers, since acquisition and extinction periods are massed. In order to degrade the predictive effect of a reinforcer, the three sequences ( $\mathrm{AE}, \mathrm{EA}$, and $\mathrm{R}$ ) were also implemented with a VI 4-min (VI 4) schedule of reinforcement replacing the $\mathrm{E}$ component. (For ease of exposition, the component in whose presence reinforcers were less frequent continued to be designated $\mathrm{E}$, although extinction was not necessarily scheduled in that component.) Thus, some reinforcers would occur during both wavelength discriminanda, thereby attenuating the predictive value of the reinforcer. Previous research indicated that similar postdiscrimination phenomena are present with VI 1 VI 4 and VI 1 EXT schedules when random stimulus sequences are used during discrimination training (Dysart, Marx, McLean, \& Nelson, 1974; cf. Guttman, 1959, and Terrace, 1968).

\section{Method}

Subjects. Twenty-four experimentally naive White Carneaux pigeons between 6 and 12 months of age and at $80 \%$ of their free-feeding weights served as subjects.

Apparatus. The experimental chambers were four identical Lehigh Valley boxes, each of which was equipped with a food hopper and a response key that could be transilluminated by light of various wavelengths. The light source was an IEE display cell containing Kodak Wratten filters $65,74,99,73$, and 72B that provided peak transmissions at $501,538,555,576$, and $606 \mathrm{~nm}$, respectively. During subsequent training, the stimulus $\left(\mathrm{S}_{1}\right)$ correlated with the schedule containing the more frequent reinforcement was $538 \mathrm{~nm}$; the stimulus $\left(\mathrm{S}_{2}\right)$ correlated with the less frequent reinforcement was $576 \mathrm{~nm}$. The chambers were illuminated by houselights whenever the response key was transilluminated. A masking noise at an intensity of $85 \mathrm{~dB}$ was continuously present during the session. Programming and recording equipment were located in an adjacent room.

Procedure. Pretraining consisted of two sessions of $15 \mathrm{~min}$ of habituation to the chamber, one session of feeder training, one session in which keypecking was shaped, and two sessions of continuous reinforcement of keypecking (35 reinforcements per session). All the subjects were then given 12 sessions of VI training. The intervals between reinforcements were randomly sampled from an exponential distribution (Fleshler \& Hoffman, 
1962) and had a mean value of $15 \mathrm{sec}$ during the 10 stimulus periods of the first VI session, $30 \mathrm{sec}$ during the 20 stimulus periods of the second VI session, and $60 \mathrm{sec}$ during the 30 stimulus periods of the remaining 10 sessions. During VI training, the response key was transilluminated with the $S_{1}$ wavelength. Each stimulus period was $60 \mathrm{sec}$ in duration and was separated from the following stimulus period by a 5 -sec blackout, during which both the keylight and houselight were off. Reinforcement consisted of $5 \mathrm{sec}$ of access to mixed grain.

Following VI training, the 24 subjects were each randomly assigned to one of six equal-sized groups that formed a $2 \times 3$ factorial design. One of two pairs of reinforcement schedules (VI 1 EXT or VI 1 VI 4) constituted the first factor, and one of the three stimulus sequences ( $\mathrm{AE}, \mathrm{EA}$, or $\mathrm{R}$ ) constituted the second factor. The AE sequence consisted of nine $S_{1}$ periods followed by nine $S_{2}$ periods. The EA sequence consisted of nine $S_{2}$ periods followed by nine $S_{1}$ periods. The $R$ sequence consisted of a quasi-random series of 32 stimulus periods containing eight $S_{1} S_{1}$ transitions and eight $S_{2} S_{2}$ transitions, with $S_{1}$ and $S_{2}$ being presented equally often. Thus the AE, EA, and $R$ sequences all contained the same number of transitions between like stimuli. Since these experiments sought to determine the effect of stimulus sequences on discrimination performance, the number of transitions between like stimuli was held constant and the number and nature of the unlike transitions were the only sequential variables that differed among groups. Groups did differ in the number of stimulus periods per session, but, since training continued until responding was stable in all groups, differences in the absolute amount of training could not alone account for differential postdiscrimination performance.

The six groups were each given 15 sessions of discrimination training, with generalization tests administered after training sessions $1,3,7,11$, and 15 . The generalization tests were conducted during extinction, with a test consisting of five presentations of each of the five wavelengths in quasi-random order. The transfer test was obtained by shifting the $\mathrm{AE}$ and EA groups to the $\mathrm{R}$ stimulus sequence for an additional eight sessions of training. The groups initially exposed to the $\mathbf{R}$ sequence were simply continued for eight sessions of further training. Generalization tests were also conducted after the fourth and eighth transfer sessions.

\section{Results}

The effects on stimulus control of the sequences present during discrimination training were assessed using measures of discrimination performance, generalization, and transfer.

Overall discrimination is described first and is followed by a presentation of the sequential effects that underlay the average rates of responding.

Figure 1 shows the mean rates (responses per minute) during $S_{1}$ and $S_{2}$ over the course of discrimination training. Some differential responding was present on the 1st day of discrimination training after nondifferential training with wavelength, and the discrimination became more pronounced as training progressed. The difference between the rates of responding during $S_{1}$ and $S_{2}$ was reliable for all groups on the 1st day ( $p<.05$ in each case) and was greater for the VI 1 EXT condition than the VI 1 VI 4 condition $[F(1,18)=8.19, \mathrm{p}<.05]$. Differential responding occurred in all sequences, but was more evident with the $R$ sequence than the AE or EA sequences. The difference between the rates of responding during $S_{1}$ and $S_{2}$ was reliably greater for the $R$ sequence than for the nonrandom sequences $[F(1,18)$

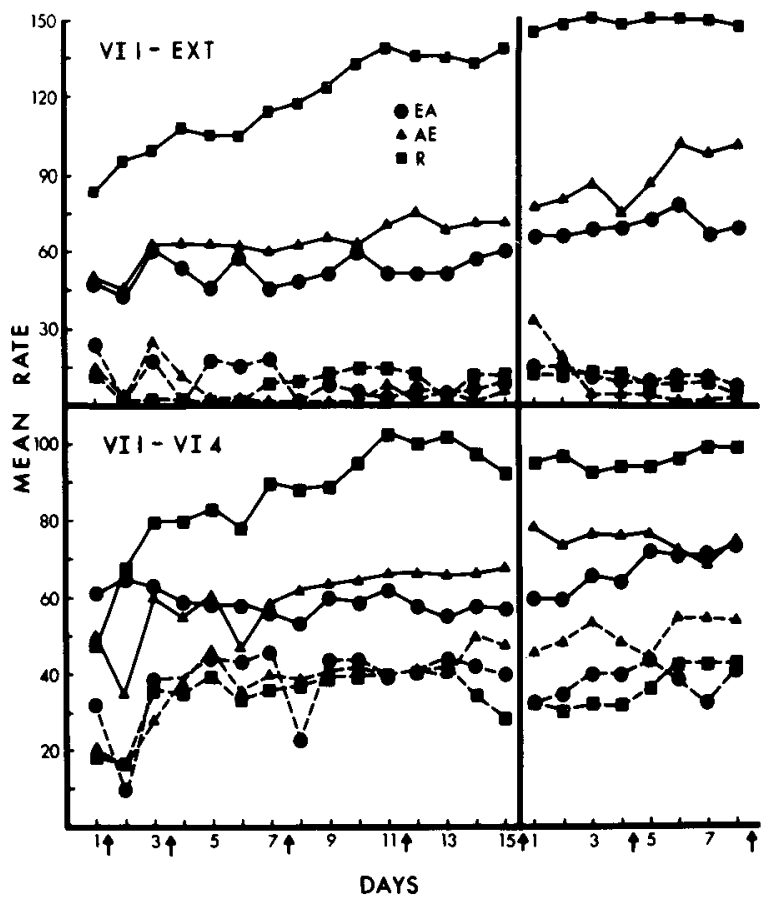

Figure 1. Mean rates of responding (responses/minute) during the discriminanda for the $\mathbf{E A}, \mathbf{A E}$, and $\mathrm{R}$ stimulus sequences. The upper panel depicts the effects of training with the VI 1 EXT reinforcement condition, and the lower panel the effects with the VI 1 VI 4 reinforcement condition. Solid lines indicate the rates during $S_{1}$, and broken lines the rates during $S_{2}$. All groups were transferred to the $R$ sequence following Day 15 . Arrows indicate the placement of generalization tests.

$=9.42, \mathrm{p}<.01] ;$ the $\mathrm{AE}$ and EA sequences did not differ from one another $(F<1)$.

Sequential effects, averaged over the 15 sessions of discrimination training with the $R$ sequence, are shown in Table 1. For both the VI 1 EXT and VI 1 VI 4 conditions, the rate of responding increased when $S_{1}$ was preceded by $S_{2}$ (a stimulus correlated with less frequent reinforcement) and decreased when $S_{2}$ was preceded by $S_{1}$ (a stimulus correlated with more frequent reinforcement). Thus, local contrast occurred. The effect of the preceding stimulus on the rate of responding to the present stimulus was statistically significant $[F(1,6)=13.24, p<.05]$ and did not vary reliably as a function of the reinforcement schedule $[F(1,6)=1.52, p>.05]$. These local contrast effects were present from the 1 st day of

Table 1

Mean Rate of Responding (in Responses per Minute) During the Prevailing Stimulus as a Function of the Preceding Stimulus

\begin{tabular}{|c|c|c|c|c|}
\hline \multirow{3}{*}{$\begin{array}{c}\text { Preceding } \\
\text { Stimulus }\end{array}$} & \multicolumn{4}{|c|}{ Reinforcement Schedules } \\
\hline & \multicolumn{2}{|c|}{ VI 1-Ext } & \multicolumn{2}{|c|}{ VI 1-VI 4} \\
\hline & $s_{1}$ & $\mathbf{s}_{2}$ & $s_{1}$ & $\mathrm{~s}_{2}$ \\
\hline $\begin{array}{l}s_{1} \\
s_{2}\end{array}$ & $\begin{array}{l}113 \\
123 \\
\end{array}$ & $\begin{array}{r}5 \\
12 \\
\end{array}$ & $\begin{array}{l}84 \\
89 \\
\end{array}$ & $\begin{array}{l}30 \\
40 \\
\end{array}$ \\
\hline
\end{tabular}


training and did not change substantially over sessions $[F(14,84)=1.04, p>.05]$.

Local contrast in the nonrandom sequences was, of necessity, confined to the one transition between $S_{1}$ and $S_{2}$ that occurred during each session (see Figure 2). The effect of the stimulus transition was most pronounced immediately following the transition, and local contrast endured for some minutes. With the $\mathrm{AE}$ sequence, responding decreased abruptly when $S_{2}$ was introduced and gradually increased thereafter. With the EA sequence, responding increased abruptly when $S_{1}$ was introduced and then decreased thereafter. The differing patterns of change following the transition in the $\mathrm{AE}$ and $\mathrm{EA}$ sequences was evidence for negative and positive contrast, respectively. The differing trends over the first $3 \mathrm{~min}$ of the transition were statistically reliable $[F(2,24)=5.25, p<.05]$. The abruptness of the change in responding upon the occurrence of the transition indicated that wavelength was sufficient to

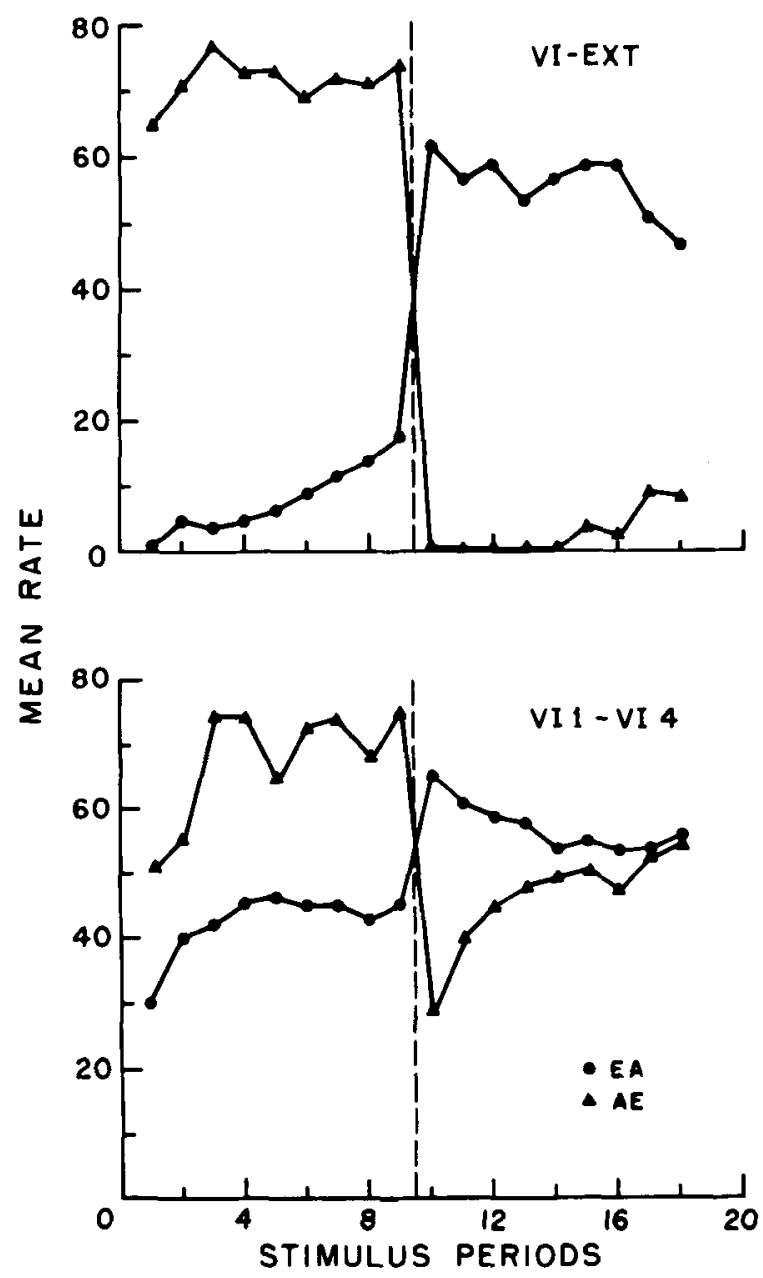

Figure 2. Mean rates of responding (responses/minute) during successive stimulus periods for the EA and AE sequences. The upper panel depicts findings with the VI 1 EXT reinforcement condition, and the lower panel findings with the VI 1 VI 4 condition. The broken vertical line indicates the point within the session at which the transition occurred betwen the two discriminanda.

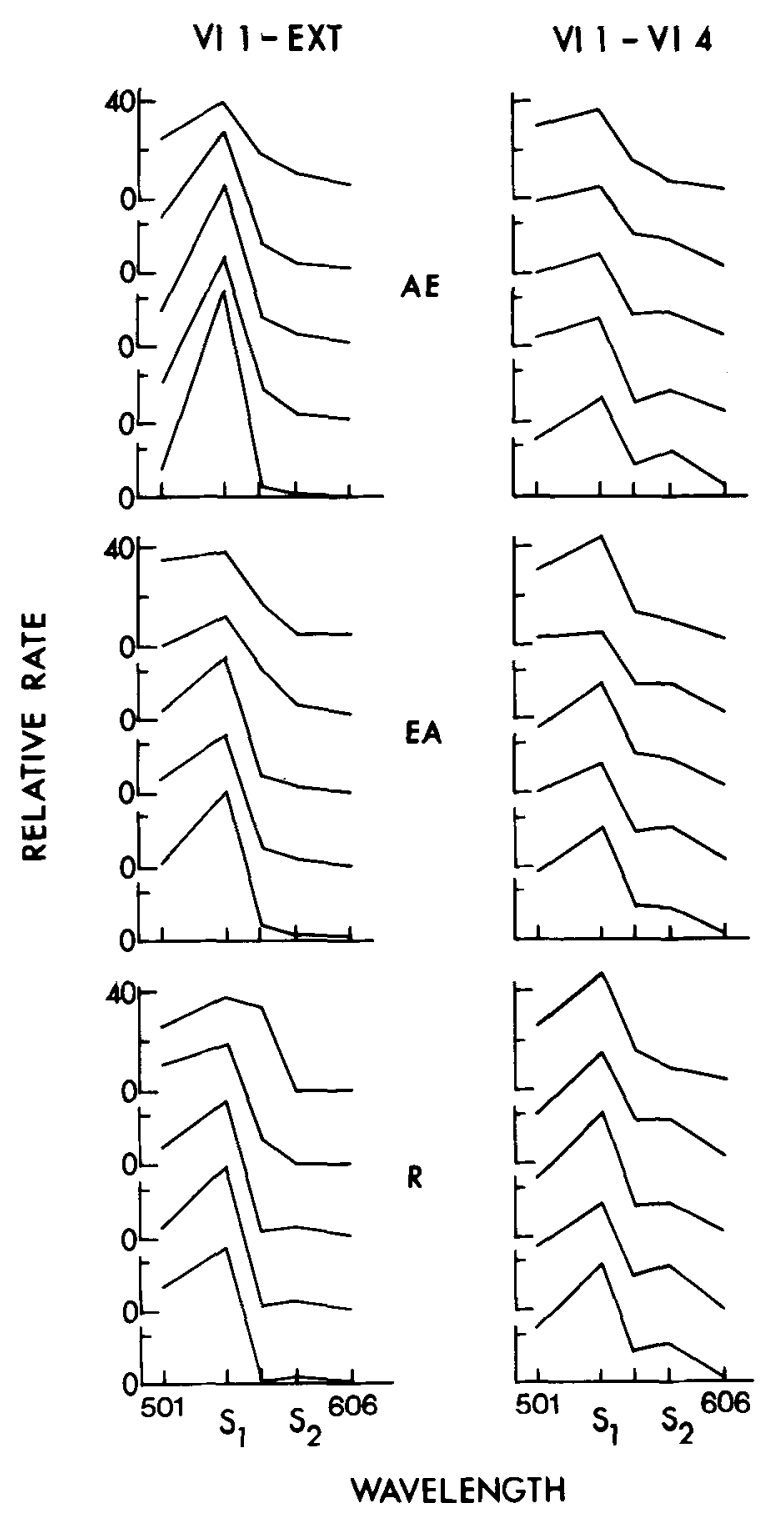

Figure 3. Relative wavelength generalization gradients following discrimination training with the $A E, E A$, and $R$ sequences. The left panel presents gradients obtained after VI 1 EXT training; the right panel presents gradients after VI 1 VI 4 training. Within each condition, the gradients, ordered from top to bottom, were obtained after $1,3,7,11$, or 15 days of training.

control responding, since the change was too prompt to be instigated solely by the often delayed change in reinforcement frequency produced by VI schedules. Thus, during discrimination training, stimulus control by the wavelength dimension was equally evident with both the $\mathrm{AE}$ and $\mathrm{EA}$ sequences. The change in the rate of responding produced by the one daily transition was reliable $[\mathrm{F}(1,12)=126.57$, $\mathrm{p}<.001$, was greater in the VI 1 EXT than the VI 1 VI 4 condition $[F(1,12)=21.10, p<.001]$, and did not differ reliably between the $\mathrm{AE}$ and $\mathrm{EA}$ sequences $[F(1,12)=1.49, p>.05]$.

Figure 3 shows the results of the five generalization tests interpolated among the training sessions. All gradients were plotted as relative rates, with the 
number of responses during a test stimulus expressed as a percentage of the total number of responses during that session.

Consider first the gradients shown in the left panel of Figure 3. These gradients were obtained from the $\mathrm{AE}, \mathrm{EA}$, and R sequences with the VI 1 EXT schedule, which replicate the procedures of the Ellis (1970) experiment except for the use of wavelength. Consistent with earlier findings, the gradients obtained after EA and $R$ training were more elevated in the region opposite to the negative stimulus than the gradients obtained after AE training. With comparable degrees of training, the $\mathrm{AE}$ gradients were uniformly steeper between $501 \mathrm{~nm}$ and $S_{1}$ than were the $E A$ and $R$ gradients. Statistical analyses indicated that the mean AE gradient, averaged over the five generalization tests, differed in shape from the combined EA and R gradients $[F(4,72)=3.17, p<.05]$ and that the shapes of the EA and $R$ gradients did not differ from one another $(F<1)$.

The gradients obtained when reinforcers were made to occur during both $S_{1}$ and $S_{2}$ through the use of a VI 1 VI 4 schedule are shown in the right panel of Figure 3. Unlike the results after VI 1 EXT training, the three sequences produced highly similar generalization gradients. All gradients tended to be bimodal in form, especially as training progressed. Ten of the 12 subjects displayed bimodality in their mean individual gradients $(p<.05)$. Statistically, there were no differential effects of stimulus sequence on the shapes of gradients following VI 1 VI 4 training $(F<1)$. Because of their bimodal form, the gradients differed in shape from those following VI 1 EXT training $[F(4,72)=5.43, p<.001]$.

Figure 1 shows transfer when all groups were shifted to the $R$ sequence. Transfer was assessed by comparing performance on the last day of original training with the first day of the $R$ sequence. With the VI 1 EXT schedule, discrimination performance following training with the $\mathrm{AE}$ sequence was disrupted, as shown by the increase in $S_{2}$ responding (see Figure 1, upper panel). Examination of individual performance within the first transfer session indicated that responding was low during the initial few $S_{2}$ periods and then gradually increased with continued exposure to the $\mathbf{R}$ sequence. Discrimination performance of the EA and $R$ groups was relatively unaffected by transfer to the $R$ sequences. Comparing the first day of transfer with the last day of original training, the differential rate of responding between $S_{1}$ and $S_{2}$ was reliably more disrupted after training with the $\mathrm{AE}$ sequence than with the $\mathrm{EA}$ and $R$ sequences $[F(1,18)=11.81, p<.01]$, while the latter two groups dis not differ from one another $(F<1)$. With the VI 1 VI 4 schedule, all stimulus sequences produced strong and equal positive transfer to the $R$ sequence (see Figure 1, lower panel). There was no reliable differential effect of stimulus sequence of transfer $(F<1)$.

Finally, with respect to performance when all groups received the $\mathbf{R}$ sequence, those subjects originally trained with the $R$ sequence continued to respond more differentially during $S_{1}$ and $S_{2}$ than those originally trained with the $\mathrm{AE}$ and $\mathrm{EA}$ sequences. This difference was reliable $[F(1,18)=8.10, p<.05]$ and was more pronounced in the VI 1 EXT than the VI 1 VI 4 condition $[F(1,18)=11.79, p<.01]$. The prior effect of stimulus sequence on the shapes of the generalization gradients after VI 1 EXT training was eliminated after transfer to the $R$ sequence. These gradients are not shown, since the interaction of gradient shape with the stimulus sequence used in original training was no longer reliable $[\mathrm{F}(4,72)=2.27$, $\mathrm{p}>.05]$.

\section{Discussion}

Earlier findings obtained with the intensity dimension (Ellis, 1970) were confirmed with the wavelength dimension when the training conditions were otherwise comparable. Following the $\mathrm{AE}$ sequence, the postdiscrimination gradient of stimulus generalization was less elevated in the region opposite to the negative stimulus and transfer to the $R$ sequence was impaired. Also consistent with previous work, the EA sequence yielded generalization gradients and transfer effects that were indistinguishable from those produced by the $\mathrm{R}$ sequence. While the differential effects of $\mathrm{AE}$ and $\mathrm{EA}$ sequences were qualitatively confirmed, the effects were quantitatively smaller with the wavelength dimension. In generalization tests, responding to the 501-nm stimulus was elevated after either EA or $\mathbf{R}$ training but did not exceed the rate during the positive stimulus $(538 \mathrm{~nm})$; that is, peak shift did not occur. A wavelength intermediate between 501 and $538 \mathrm{~nm}$ would likely have controlled the anticipated high rate. In transfer to the $R$ sequence, discrimination performance was disproportionately disrupted following AE training, but differential responding was not eliminated as it had been with the intensity dimension. The smaller disruption of transfer was probably due to the more precise stimulus control produced by nondifferential training with the wavelength dimension. In support of this interpretation, a reliable difference was present between the rate of responding during $S_{1}$ and $S_{2}$ within the first session of discrimination training with the wavelength dimension but not with the intensity dimension (Ellis, 1970).

Differential sequence effects were eliminated when a discrimination procedure was used in which reinforcers could occur during both discriminanda. With the VI 1 VI 4 schedule, the $\mathrm{AE}$ sequence led to generalization and transfer that were essentially the same as those found with the EA and $R$ sequences. The prospect is therefore raised that the differential se- 
quence effects found in the Ellis (1970) experiment, and possibly elsewhere (Honig, Thomas, \& Guttman, 1959; Rosen \& Terrace, 1975), were due to the introduction of a new variable through the use of nonrandom sequences rather than to the uncovering of a variable that was inherent within the random sequences. A likely candidate for this new variable is the discriminative function of reinforcement, a function that cannot develop with random sequences of discriminanda.

The precise contribution of the discriminative effects of reinforcement to differential sequence effects cannot be a simple one, however. First, during discrimination training, there is clear evidence that the wavelength discriminanda were sufficient to control differential responding independently of any possible discriminative effect of reinforcement. This was shown by the immediate change in the rate of responding upon the transition between the discriminanda in both the $\mathrm{AE}$ and EA sequences (see Figure 2). The change occurred prior to a discriminably long period without reinforcement in the $\mathrm{AE}$ sequence or prior to the first occurrence of reinforcement in the EA sequence. Second, reinforcers are potentially capable of acquiring a discriminative function with both $\mathrm{AE}$ and EA sequences when a VI 1 EXT schedule is used, and yet alterations in generalization and transfer occurred only with the AE sequence. Therefore, some interaction between the stimulus conditions prevailing during training and the stimulus conditions prevailing during generalization and transfer must have produced the observed differential sequence effects.

Although speculative, the following analysis of the stimulus conditions present first during training and then during testing provides a possible interpretation of differential sequence effects. During training with the $\mathrm{AE}$ sequence, reinforcement occurred toward the beginning of the session and provided the context in which both responding and the transition to $S_{2}$ occurred. During training with the EA sequence, however, nonreinforcement was present toward the beginning of the session and served as the context for both responding and the transition to $S_{1}$.

Consider now the stimuli present during generalization testing and their similarity to those in the training conditions. During generalization, nonreinforcement was continuously present, since the test was conducted during extinction. Nonreinforcement thus provided the context in which all stimulus transitions took place. Therefore, during generalization testing, both the nonreinforced context and the relationship of that context to stimulus transitions more closely duplicated the conditions present during training with the EA than with the $\mathrm{AE}$ sequence. Consistent with an analysis in which behavior is controlled by stimuli in addition to wavelength, appreciable responding occurred to stimuli other than $S_{1}$ after training with the EA sequence. After $\mathrm{AE}$ training, however, responding was more narrowly confined to $S_{1}$, the stimulus from the AE sequence that was present both in training prior to reinforced responding and in generalization testing.

Turning to the stimuli present during the transfer test, reinforcement occurred at the beginning of exposure to the $\mathbf{R}$ sequence, to which all groups were shifted, and transitions occurred after both reinforcement and nonreinforcement. Since reinforcers were present at the beginning of the transfer test, stimulus control by wavelength after the $\mathrm{AE}$ sequence would be favored over that after the EA sequence. The occurrence of transitions after both reinforcement and nonreinforcement would not differentially favor either nonrandom sequence. The net effect of these two factors might be thought, therefore, to tend to favor transfer from the $\mathrm{AE}$ to the $\mathrm{R}$ sequence. However, within the first session of transfer from the $\mathrm{AE}$ to the $\mathbf{R}$ sequence, differential responding to the wavelength discriminanda deteriorated, with the result that, over the entire session, stimulus control by wavelength was poorer following $\mathrm{AE}$ than following EA training. The following interpretation of this result is given: Since the VI 1 EXT schedule permitted the reinforcer to acquire a discriminative function and since reinforcers were previously present at the beginning of both the training and transfer session for only the $\mathrm{AE}$ sequence, responding during $\mathrm{S}_{\mathbf{2}}$ increased in the transfer test as the discriminative function of the reinforcer progressively overshadowed control by wavelength as the transfer test continued. There is ample evidence that reinforcers can function as salient discriminative stimuli (e.g., Rilling \& Howard, 1981) and that such stimuli are effective in controlling responding (e.g., Capaldi, 1971).

Whatever the ultimate validity of the foregoing speculations, the central point to be reemphasized is that differential effects of $\mathrm{AE}$ and $\mathrm{EA}$ training sequences on generalization and transfer probably tell us less about general issues in the acquisition of stimulus control than they do about the specific discriminative effects of reinforcers with nonrandom sequences of the nominal discriminative stimuli. As such, it is problematic whether the manipulation of stimulus sequences is well suited to uncover the fundamental behavioral processes underlying the acquisition of stimulus control with random sequences (cf. Rosen \& Terrace, 1975). It appears that nonrandom sequences of discriminative stimuli introduce new processes, such as the discriminative function of reinforcers, rather than merely make explicit processes that are inherent within random sequences.

The conclusion that the EA sequence is not necessary for the occurrence of various postdiscrimination phenomena was based upon the presence of comparable generalization and transfer with the $A E$ and $R$ sequences using a VI 1 VI 4 reinforcement schedule. By scheduling reinforcers during $S_{2}$, the discriminative function of the reinforcer was degraded and competi- 
tion with the nominal discriminative stimuli was presumably lessened. This conclusion must be tempered, with respect to stimulus control in the generalization test, by the presence of bimodal gradients rather than by gradients that were elevated in the region removed from $S_{2}$ and depressed in the vicinity of $S_{2}$. Gradients of this latter form have previously been reported following training with a random sequence in which unequal VI schedules were correlated with the discriminanda (Dysart et al., 1974; Guttman, 1959; Terrace, 1968). In this other work, however, the wavelength spacing between $S_{1}$ and $S_{2}$ was less $(20 \mathrm{~nm})$ than that used in the present experiment $(38 \mathrm{~nm})$. Bimodal gradients have been found following training with equal reinforcement frequencies during $S_{1}$ and $S_{2}$ (Kalish \& Guttman, 1957) and with maintained generalization procedures (Blough, 1969). It is clear, however, that a more straightforward case could be made for the absence of differential sequence effects if the discriminative effects of the reinforcer were degraded by a procedure that also yielded typical postdiscrimination phenomena. This was a major purpose of the second experiment.

\section{EXPERIMENT 2}

Experiment 2 continued to pursue the possibility that differential sequence effects were due to the reinforcer's having acquired a discriminative function with nonrandom stimulus sequences rather than to sequences having a fundamental role in discrimination formation. To strengthen the implications of the present experiment for the interpretation of the original Ellis (1970) study of sequence effects, the putative discriminative function of reinforcement was manipulated in a task in which the discriminanda varied in visual intensity.

In Experiment 1, the potential discriminative function of the reinforcer was degraded by occasionally delivering reinforcers during the stimulus correlated with the lower reinforcement density. In Experiment 2, a different procedure-the variable-trials (VT) procedure described below-in which the discriminative effect of the reinforcer was degraded by occasionally omitting reinforcers during the stimulus $(\mathrm{S}+)$ correlated with reinforcement was instituted. Reinforcers never occurred during the stimulus (S-) correlated with extinction. By means of the VT procedure, complications produced by bimodal generalization gradients were avoided and the likelihood of obtaining more typical postdiscrimination findings with the $R$ sequence was enhanced.

Heretofore, the discriminative function of the reinforcer between stimulus periods was considered. With nonrandom sequences of the nominal discriminanda, the reinforcing stimulus may acquire a discriminative function with respect to responding during subsequent stimulus periods. This between-periods source of stimulus control is possible because the $S+$ periods are massed in $\mathrm{AE}$ and $\mathrm{EA}$ sequences. With freeoperant procedures, however, there is a second means whereby the reinforcer may acquire a discriminative function. Since multiple reinforcers often occur within a single stimulus period, the reinforcer may serve as a discriminative stimulus for continued responding within the remainder of that same period (Jenkins, 1965). The within-periods origin of a discriminative function for the reinforcing stimulus apparently plays little role with random sequences of the discriminanda (Pierrel \& Blue, 1967; Razlear, PierrelSorrentino, \& Brissey, 1975), but it could potentially affect performance with nonrandom sequences. Through the use of the VT procedure, the withinperiods discriminative function of the reinforcer was eliminated in Experiment 2 and the between-periods discriminative function was manipulated.

\section{Method}

Subjects. Twenty-four experimentally naive pigeons maintained at $80 \%$ of their free-feeding weights served as subjects.

Apparatus. The four experimental chambers from Experiment 1 were used with neutral density filters, rather than chromatic filters, inserted in the IEE display cells. The neutral density filters provided white light at the same intensities that had been used previously by Ellis (1970): 23.7, 38.7, 60.2, 95.8, 153, 242, and $383 \mathrm{~lx}$. The S+ intensity was $95.8 \mathrm{~lx}$; the S- intensity was $242 \mathrm{~lx}$.

Procedure. The pretraining method was identical to that used in Experiment 1 except for the institution of a VT procedure. For the VT procedure, the length of each stimulus period was determined by the same exponential distribution used to determine the interreinforcement intervals in Experiment 1. However, reinforcers, if any, occurred only after the last response in the stimulus period. For a stimulus period in which a reinforcer was available, the reinforcer occurred if a response was emitted within $10 \mathrm{sec}$ of the programmed duration of the period. Essentially all scheduled reinforcers were collected with this criterion. The procedure eliminated the discriminative function of reinforcement within periods, since the reinforcer could occur only at the end of the stimulus period. A 5-sec blackout interval separated all stimulus periods. It is important to note that the VT procedure maintained the same average duration of stimulus periods and the same average interreinforcement intervals as were present in the Ellis (1970) investigation and in Experiment 1 of this report. Thus, the discriminative function of reinforcement was varied without grossly altering the conditions under which differential sequence effects had previously been observed.

Following pretraining with a mean $S+$ duration of $60 \mathrm{sec}$, the 24 subjects were assigned to one of six groups that formed a $2 \times 3$ factorial design. The factor having three levels was the stimulus sequence: $\mathrm{AE}, \mathrm{EA}$, or $\mathrm{R}$. The factor having two levels was the percentage of $S+$ trials that terminated with a reinforcer: $100 \%$ or $50 \%$. By reducing the percentage of reinforced trials to $50 \%$, the potential discriminative function of the reinforcer between stimulus periods was degraded in the $\mathrm{AE}$ and $\mathrm{EA}$ sequences, since an $\mathrm{S}+$ period was then preceded by a reinforcer on only half the trials. The number of stimulus periods making up each daily training session was the same as that in Experiment 1, with the transitions between like stimuli equated for all groups. The number of training sessions, the placement of generalization tests, and the number of transfer sessions were also identical to those used in Experiment 1. During the generalization tests, extinction was scheduled with each of the seven intensities being presented in quasi-random order within six successive blocks. 
Results and Discussion

The effects of the AE, EA, and $R$ sequences on stimulus control after training with the VT procedure were examined during discrimination, generalization, and transfer.

The mean rates (responses per minute) during $\mathrm{S}+$ and $S-$ are shown in Figure 4. For both the $100 \%$ (upper panel) and $50 \%$ (lower panel) reinforcement conditions, the rates during $\mathrm{S}+$ and $\mathrm{S}-$ were similar on the 1st day of discrimination training and progressively diverged as training continued. Thus, as anticipated, nondifferential pretraining produced less stimulus control by the intensity dimension than was previously obtained with wavelength. Statistical evaluation indicated that none of the groups differed in their responding during $S+$ and $S-$ on the 1st day of training ( $p>.05$ in each case) and that the rates diverged thereafter $[F(14,252)=23.31, p<.001]$. By the end of training, differential responding during $\mathrm{S}+$ and $\mathrm{S}$ - was greater with the $\mathbf{R}$ sequence than with the nonrandom sequences, and with the $\mathrm{AE}$ than with the EA sequence. The pattern of results was similar in both the $100 \%$ and $50 \%$ reinforcement conditions. As evaluated over the last 3 days of training, the difference between the rates of responding during $\mathrm{S}+$ and $\mathrm{S}$ - was reliably greater in the $\mathbf{R}$ than in the nonrandom sequences $[F(1,18)=7.32, p<.05]$,

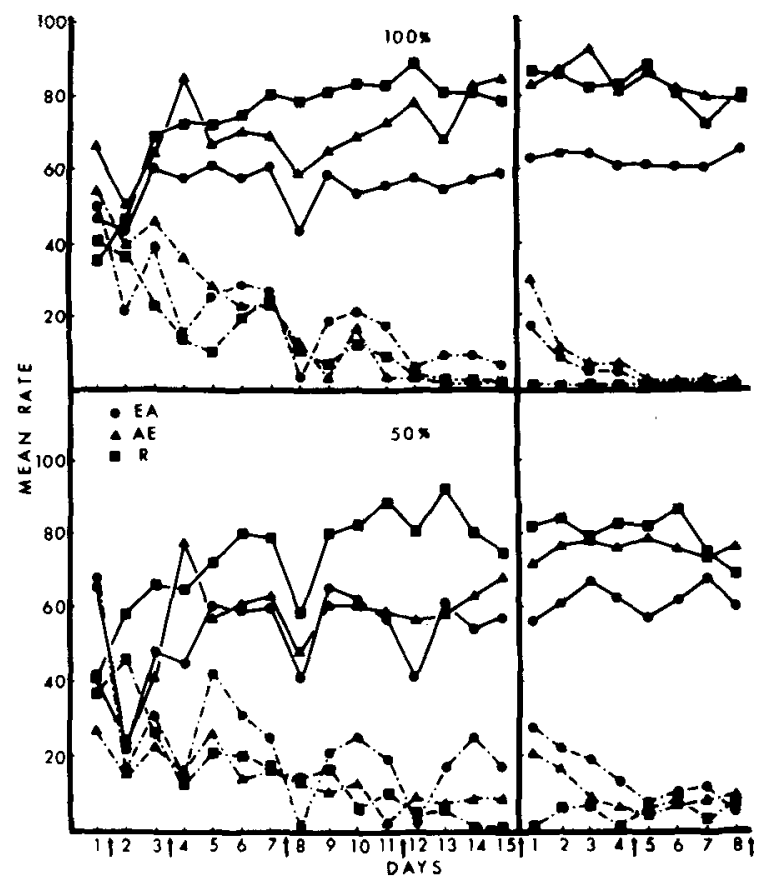

Figure 4. Mean rates of responding (responses/minute) during the discriminanda for the EA, AE, and $R$ stimulus sequences. The upper panel depicts the effects of training with reinforcement at the termination of $100 \%$ of the S+ trials, and the lower panel the effects with reinforcement at the termination of $50 \%$ of the S+ trials. Solid lines indicate the rates during $\mathrm{S}+$, and broken lines the rates during $\mathbf{S}-$. All groups were transferred to the $\mathbf{R}$ sequence following Day 15. Arrows indicate the placement of generalization tests.
Table 2

Mean Rate of Responding (in Responses per Minute) During the Prevailing Stimulus as a Function of the Preceding Stimulus

\begin{tabular}{cccccc} 
& \multicolumn{4}{c}{ Reinforcement Schedules } \\
\cline { 2 - 5 } Preceding & \multicolumn{2}{c}{$100 \%$} & & \multicolumn{2}{c}{$50 \%$} \\
\cline { 2 - 5 } Stimulus & $\mathrm{s}_{1}$ & $\mathrm{~s}_{2}$ & & $\mathrm{~s}_{1}$ & $\mathrm{~s}_{2}$ \\
\hline $\mathrm{s}_{1}$ & 70 & 1 & 71 & 1 \\
$\mathrm{~s}_{2}$ & 86 & 4 & 91 & 4 \\
\hline
\end{tabular}

was greater in the $A E$ than in the $\mathrm{EA}$ sequence $[F(1,18)$ $=5.02, p<.05]$, and was unaffected by the percentage of reinforced $S+$ trials $[F(1,18)=1.51, p>.05]$.

Sequential effects occurred during discrimination training with the VT procedure essentially as they had with the standard free-operant procedure of Experiment 1. Within the $R$ sequence, in which there were many transitions between the two discriminanda, both positive and negative local contrast occurred. As shown in Table 2, the rate of responding, averaged over the 15 sessions of discrimination training, was decreased when $\mathrm{S}$ - was preceded by $\mathrm{S}+$ and was increased when $\mathrm{S}+$ was preceded by $\mathrm{S}-$. The magnitude of local contrast was unaffected by the percentage of reinforcement. The differential effects of the previous stimulus on the rate of responding to the prevailing stimulus were reliable $[F(1,6)=20.67$, $\mathrm{p}<.01]$ and did not interact with the percentage of $\mathrm{S}+$ reinforcement $(\mathrm{F}<1)$. Although differential responding during the discriminanda did not develop within the first session of training, sequence effects were already present and the magnitude of local contrast did not change reliably over the 15 training sessions $(F<1)$. Local contrast also occurred with the $\mathrm{AE}$ and $\mathrm{EA}$ sequences, as in Experiment 1. (The data are not displayed because they were essentially equivalent to those shown earlier; cf. Figure 2.) Responding in the AE sequence immediately decreased upon the transitions to S- and then recovered somewhat. Responding in the EA sequence immediately increased upon the transition to $\mathrm{S}+$ and declined thereafter. Thus stimulus control by the discriminanda was again clearly present during training sessions with the nonrandom sequences. As in Experiment 1 , these differing trends produced a reliable interaction between stimulus sequences over the initial three stimulus periods following the transition $[F(2,24)=3.54, p<.05]$.

The gradients obtained from the various experimental conditions are examined first. Then internal evidence regarding the effect of the discriminative function of reinforcement on responding during generalization testing is presented.

Figure 5 contains the relative generalization gradients obtained from the five tests conducted during discrimination training. Unlike those obtained with the standard free-operant procedure, the AE and EA gradients were highly similar in shape. For all three 


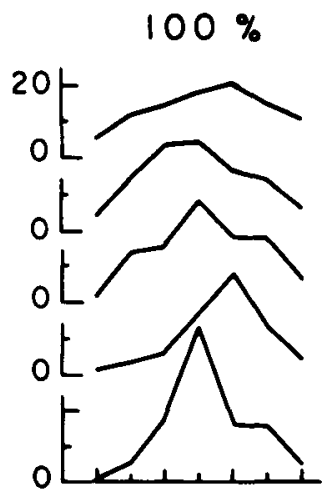

$\mathbf{A E}$
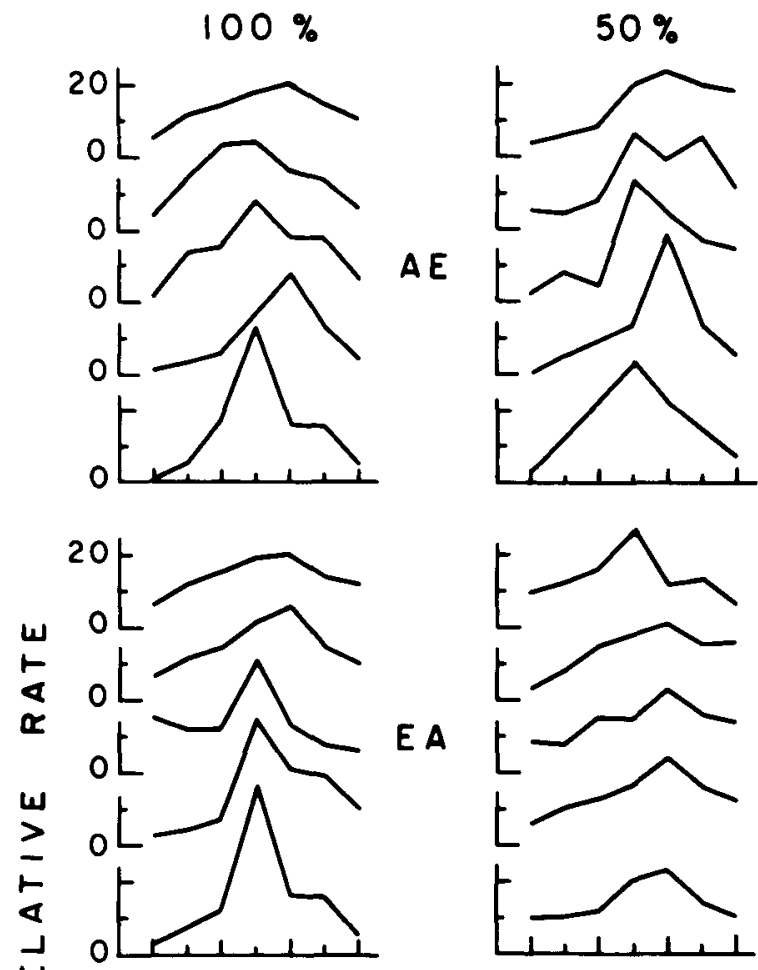

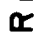
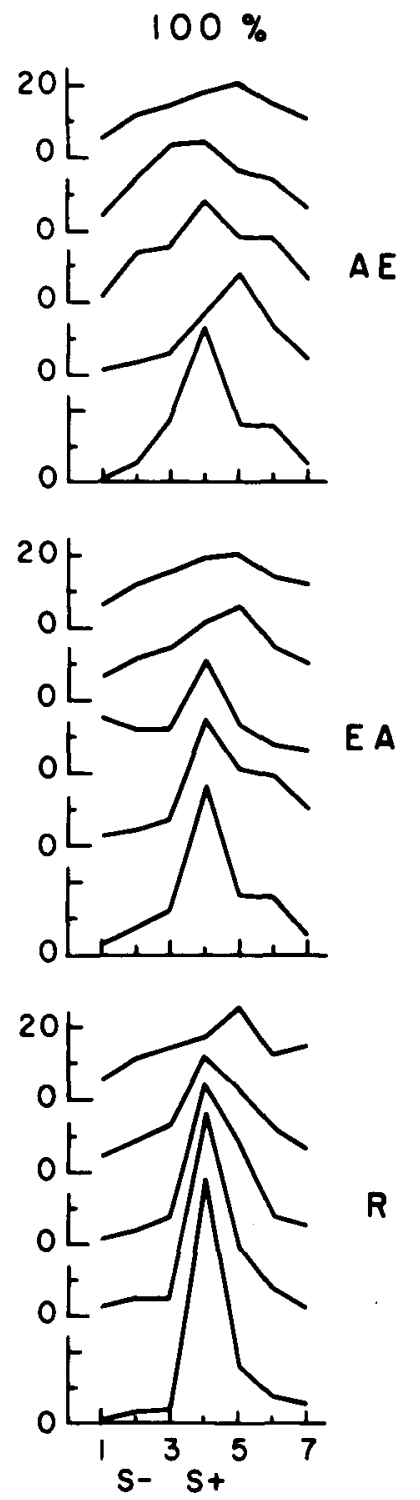

E A

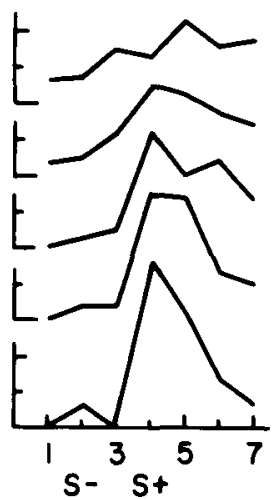

INTENSITY

Figure 5. Relative intensity generalization gradients following discrimination training with the $\mathbf{A E}, \mathbf{E A}$, and $\mathbf{R}$ sequences. The left panel depicts gradients obtained after training with $100 \%$ reinforcement at the termination of S+ trials; the right panel depicts gradients after training with $50 \%$ reinforcement at the termination of S+ trials. Within each condition, the gradients, ordered from top to bottom, were obtained after $1,3,7,11$, or 15 days of training.

sequences and both reinforcement conditions, the generalization gradients were elevated in the region removed from $\mathrm{S}-$, with peak shift occurring in a number of the tests. Thus, with the VT procedure, typical postdiscrimination findings were obtained. With the $\mathbf{R}$ sequence, in which multiple transitions occurred between $\mathrm{S}+$ and $\mathrm{S}-$, the gradients were steeper than those found after training with nonrandom sequences. No statistically reliable differences occurred between the shapes of the AE and EA gradients $(F<1)$, and these gradients differed as a set from those obtained after the $R$ sequence $[F(6,108)$
$=3.55, \mathrm{p}<.01]$. When the five generalization tests were averaged for all sequences taken together, the mean gradient obtained after training with $100 \%$ reinforcement was steeper than that after $50 \%$ reinforcement $[F(6,108)=2.65, p<.05]$.

As an indication of whether the reinforcing stimulus had acquired a discriminative function, the course of responding within each generalization test was examined. As a measure of resistance to extinction, the rate of responding was determined in six successive blocks of stimulus periods within each generalization test. Within each block, each of the seven test stimuli was presented once. As shown in Figure 6, responding declined within the generalization tests after $\mathrm{AE}$ train-
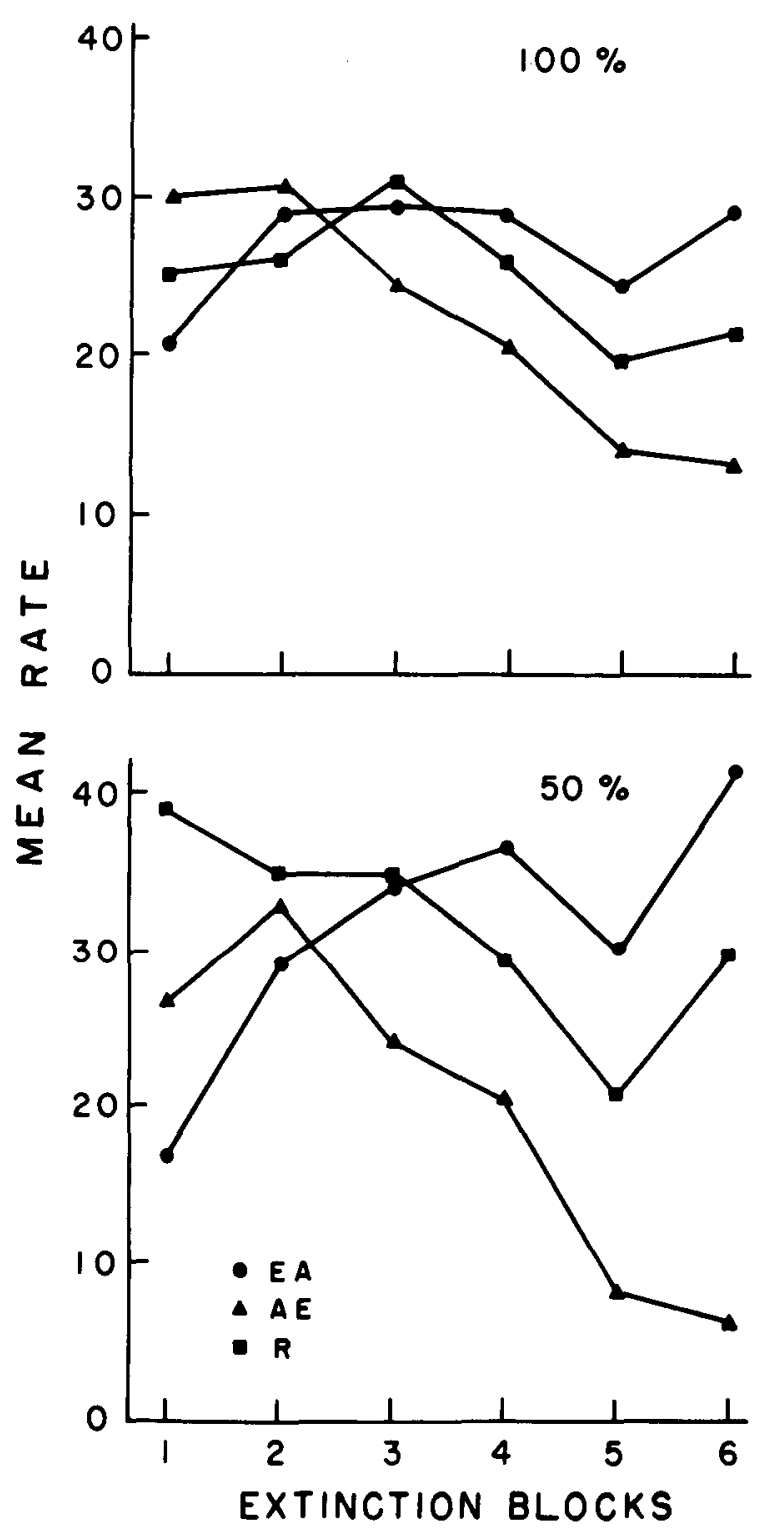

Figure 6. Mean rates of responding (responses/minute) across successive extinction blocks of generalization testing following training with the $E A, A E$, and $R$ sequences. The upper panel depicts the findings after reinforcement at the termination of $100 \%$ of the $S+$ trials, and the lower panel the findings after reinforcement at the termination of $50 \%$ of the $S+$ trials. 
ing (in which nonreinforcement had never been followed by reinforcement) and increased after EA training (in which nonreinforcement had always been followed by reinforcement). The course of responding during extinction was reliably different for the $\mathrm{AE}$ and $\mathrm{EA}$ sequences $[\mathrm{F}(5,90)=9.41, \mathrm{p}<.05]$ and was unaffected by the percentage of reinforcement $(F<1)$. After training with the $R$ sequence (in which nonreinforcement had been followed by both $S+$ and $\mathrm{S}-$ ), responding remained relatively constant within the test session. There was no statistically reliable effect of extinction blocks on the rate of responding during generalization after the $R$ sequence $[F(5,90)$ $=1.65, \mathrm{p}>.05]$.

As in Experiment 1, transfer of stimulus control was assessed by comparing discrimination on the last day of original training with the initial day in which all subjects were exposed to the $R$ sequence (see Figure 4). Consider first the $100 \%$ reinforcement condition that eliminated the within-periods contribution to the discriminative function of reinforcement, but in which the between-periods contribution remained intact. As shown in the upper panel of Figure 4, differential responding continued to be somewhat disrupted upon transfer to the $\mathrm{R}$ sequence, with the disruption greater after the AE than after the EA sequence. Once again, an examination of individual data indicated that disruption within the first transfer session after $\mathrm{AE}$ training was primarily due to a progressive increase in the rate of responding during $S-$ as the session continued. Although small in absolute terms, the consistency of individual performance yielded a reliably greater disruption of transfer following the $\mathrm{AE}$ than following the $\mathrm{EA}$ sequence $[\mathrm{F}(1,18)=10.57$, $\mathrm{p}<.05]$.

Consider now the $50 \%$ reinforcement condition, in which the putative discriminative function of the reinforcer was degraded between stimulus periods. As shown in the lower panel of Figure 4, although there was some general disruption following transfer from the nonrandom sequences to the $R$ sequence, there was no differential disruption by the $A E$ and EA sequences. The absence of a differential transfer effect on stimulus sequences in the $50 \%$ reinforcement condition ( $F<1)$ and the earlier reported presence of a differential effect in the $100 \%$ condition resulted in a significant interaction $[F(1,12)=5.31$, $\mathrm{p}<.05]$.

Upon continued exposure of all subjects to the $\mathbf{R}$ sequence, group differences disappeared in discrimination performance and stimulus generalization. As measured during the last three sessions of transfer to the $R$ sequence, equivalent differential responding was achieved by the subjects previously exposed to different stimulus sequences in original training $[F(2,18)=1.23, p>.05]$. As measured during the two generalization tests interspersed during transfer to the $\mathbf{R}$ sequence, the relative gradients for all groups were similarly shaped (i.e., elevated in the region opposite to $\mathrm{S}-$ ) and responding across extinction blocks within each test was comparable (i.e., relatively constant). No reliable interaction of gradient shape $(F<1)$ or extinction blocks $[F(10,90)=1.40$, $p>.05]$ with stimulus sequences was obtained after all subjects had been transferred to the $R$ sequence.

\section{GENERAL DISCUSSION}

In both Experiments 1 and 2, when procedures were instituted to reduce the discriminative function of the reinforcer, the differential sequence effects reported by Ellis (1970) disappeared. The differential effects of $\mathrm{AE}$ and $\mathrm{EA}$ sequences on generalization and transfer were eliminated with the wavelength dimension (Experiment 1) and the intensity dimension used by Ellis (Experiment 2) when the discriminative function was degraded either by adding reinforcers during the negative stimulus (Experiment 1) or by omitting reinforcers during the positive stimulus (Experiment 2). The most parsimonious integration of the present findings with those of Ellis (1970) is that, with the more typical random sequences of discriminanda, differential sequence effects do not fundamentally contribute to the acquisition of stimulus control, and that the observed sequence effects arose from the opportunity for reinforcement to acquire a discriminative function with nonrandom training sequences. As such, nonrandom sequences of the discriminanda are probably not well suited for the illumination of the behavioral processes found with random training sequences (cf. Ellis, 1970; Hinson \& Malone, 1980; Rosen \& Terrace, 1975).

The precise means whereby the discriminative function of the reinforcing stimulus interacted with the other stimuli controlling performance during generalization and transfer tests was incompletely specified by these experiments. The task of analyzing this interaction is not a compelling one, however, since the outcome of the enterprise would likely reveal less about the general processes essential for discrimination formation than about the specific processes generated by nonrandom sequences of the nominal discriminanda. An understanding of the fundamental processes of discrimination formation apparently does not force an analysis of these types of sequence effects.

Although the analysis of sequential effects is incomplete, some tentative conclusions may be reached. First, there is substantial indirect evidence that nonrandom training sequences do, in fact, lead to a discriminative function for the reinforcer. During generalization testing, the course of extinction was related in an orderly manner to the type of training sequence (Experiment 2), and, during the transfer 
test, responding in the negative stimulus actually increased within the first session of exposure to the random sequence (Experiments 1 and 2). Second, a within-periods discriminative function of the reinforcer may be necessary, but it is not sufficient to produce a differential effect of $\mathrm{AE}$ and $\mathrm{EA}$ sequences on generalization. That a within-periods discriminative function may be necessary is shown by the absence of differential sequence effects on generalization gradients following training with the VT procedure (Experiment 2) and its presence in the free-operant procedures of Experiment 1 and Ellis (1970). That a within-periods discriminative function is not sufficient is suggested by the absence of differential sequence effects when reinforcers were scheduled during the negative stimulus using a free-operant procedure (Experiment 1). Thus, the opportunity for the reinforcer to acquire a between-periods discriminative function may first need to exist before an effect of a withinperiods discrimination function is observed. Third, a between-periods discriminative function of reinforcement may be both necessary and sufficient to produce a differential effect of $\mathrm{AE}$ and $\mathrm{EA}$ sequences on transfer to a random sequence of discriminanda. That the between-periods discriminative function may be necessary is shown by the occurrence of differential transfer if, and only if, the predictive value of the reinforcer was not degraded (Ellis, 1970; Experiment 1, in the VI 1 EXT condition; Experiment 2, in the $100 \%$ reinforcement condition). That the between-periods discriminative function may be sufficient is shown by the presence of differential transfer when the within-periods discriminative function of reinforcement was eliminated (Experiment 2, in the $100 \%$ reinforcement condition). Thus, the differential sequence effect on transfer appears to be solely a consequence of the between-periods discriminative function of reinforcement, whereas the differential effect on generalization may involve both the between- and within-periods discriminative functions. These conclusions on the sources of differential sequence effects in generalization and transfer must remain tentative, since the various experiments may differ in important respects as yet unrecognized, although the general characteristics of the present studies and of Ellis (1970) are highly similar.

Although stimulus sequences during training apparently do not contribute fundamentally to discrimination formation, there is ample evidence that they do affect the absolute rate of responding. During discrimination training, transitions between successive discriminanda increased the magnitude of differential responding to the second stimulus. Such local effects on the absolute strength of responding are labeled behavioral contrast in the operant procedure (Skinner, 1938). With some free-operant procedures, local contrast effects may contribute to a sustained elevation of responding during the positive discriminative stimulus through the adventitious reinforcement of high rates of responding (e.g., Marcucella \& MacDonall, 1977).

A second contribution of stimulus sequences to the absolute rate of responding occurred during generalization testing when extinction was scheduled. As the test session progressed, the $\mathrm{AE}$ sequence generated a decreasing rate, the EA sequence an increasing rate, and the $R$ sequence a constant rate of responding. These differing trends suggest that reinforcement and nonreinforcement provided a part of the stimulus context that controlled responding and that responding during extinction was therefore appropriate to the stimulus conditions present during training. Consistent with this interpretation, similar effects of nonrandom sequences of reinforcement and nonreinforcement on resistance to extinction have been extensively documented with discrete-trial procedures (e.g., Capaldi, 1966, 1971).

Most generally, the present findings highlight the conceptual difficulties that may be engendered when the outcome of a differential conditioning procedure is loosely described as acquiring a "discrimination." Technically, what has been acquired is a change in the stimulus control of behavior. To label that change a "discrimination" is to risk reifying the effects of the procedure that produced the change. The extent to which the change in stimulus control is manifest in a given test environment depends upon the degree to which the test environment contains all, and only all, of the controlling stimuli present in the training environment. The test environment does not simply call forth an invariant entity called a "discrimination."

The foregoing discussion parallels, of course, Skinner's treatment of discrimination (Skinner, 1938, p. 229) and of concepts (Skinner, 1977). In the current instance, different stimulus sequences during training led to differing stimulus control in generalization and transfer tests only to the extent that the training procedures permitted differences in the discriminative effect of reinforcement to affect test performance. By employing a VT procedure with $50 \%$ reinforcement, the within-periods and between-periods origins of the discriminative function of reinforcement may be eliminated and reduced, respectively. It is of some interest to note that the original free-operant procedures devised to study stimulus control typically confined the reinforcer to the final portion of the stimulus period (Ferster \& Skinner, 1957, p. 526), thereby preventing the reinforcer from acquiring a discriminative function within periods. The VT procedure has much to recommend it, since the procedure avoids the discriminative function of reinforcement within stimulus periods, as does the discrete-trials procedure, while retaining the reliability and high resistance to extinction characteristic of the typical free-operant procedure. 


\section{REFERENCES}

Blovgh, D. S. Generalization gradient shape and summation in steady-state tests. Journal of the Experimental Analysis of Behavior, 1969, 12, 91-104.

Blovgr, D. S. Steady state data and a quantitative model of operant generalization and discrimination. Journal of Experimental Psychology: Animal Behavior Processes, 1975, 104, 3-21.

Capaldi, E. J. Partial reinforcement: An hypothesis of sequential effects. Psychological Review, 1966, 73, 459-477.

Capaldi, E. J. Memory and learning: A sequential viewpoint. In W. K. Honig \& P. H. R. James (Eds.), Animal memory. New York: Academic Press, 1971.

Donahoe, J. W., McCroskery, J. H., \& Richardson, W. K. Effects of context on the postdiscrimination gradient of stimulus generalization. Journal of Experimental Psychology, 1970, 84, 58-63.

Dysart, J., Marx, M. H., Mclean, J., \& Nelson, J. A. Peak shift as a function of multiple schedules of reinforcement. Journal of the Experimental Analysis of Behavior, 1974, 22, 463-470.

ELLIs, W. R. Role of stimulus sequences in stimulus discrimination and stimulus generalization. Journal of Experimental Psychology, 1970, 83, 155-163.

Essock, S. M., \& Blough, D. S. Effects of stimulus spacing on steady state gradients of inhibitory control. Animal Learning \& Behavior, 1977, 5, 174-176.

Ferster, C. B., \& Skinnen, B. F. Schedules of reinforcement. Englewood Cliffs, N.J: Prentice-Hall, 1957.

Fleshler, M., \& Hoffman, H. S. A progression for generating variable-interval schedules. Journal of the Experimental Analysis of Behavior, 1962, 5, 529-530.

GutTman, N. Generalization gradients around stimuli associated with different reinforcement schedules. Journal of Experimental Psychology, 1959, 58, 335-340.

Hanson, H. M. Effects of discrimination training on stimulus generalization. Journal of Experimental Psychology, 1959, 58, 321-334.

Hinson, J. M., \& Malone, J. C., JR. Local contrast and maintained generalization. Journal of the Experimental Analysis of Behavior, 1980, 34, 263-272.

Honic, W. K., Thomas, D. R., \& Gutrman, N. Differential effects of continuous extinction and discrimination training on the generalization gradient. Journal of Experimental Psychology, 1959, 58, 145-152.

Jenkins, H. M. Measurement of stimulus control during discriminative operant conditioning. Psychological Bulletin, 1965, 64, 365-376.

Kalish, H. I., \& Guttman, N. Stimulus generalization after equal training on two stimuli. Journal of Experimental Psychology, 1957, 53, 139-144.

Malone, J. C., JR., \& Staddon, J. E. R. Contrast effects in maintained generalization gradients. Journal of the Experimental Analysis of Behavior, 1973, 19, 167-180.

Marcucella, H., \& MacDonall, J. S. A molecular analysis of multiple schedule interactions: Negative contrast. Journal of the Experimental Analysis of Behavior, 1977, 28, 71-82.

Nevin, J. A., \& Shetrleworth, S. J. An analysis of contrast effects in multiple schedules. Journal of the Experimental Analysis of Behavior, 1966, 9, 305-315.

Pierrel, R., \& BlUE, S. Antecedent reinforcement contingencies in the stimulus control of an auditory discrimination. Journal of the Experimental Analysis of Behavior, 1967, 10, 545-550.

Razlear, T. G., Pierrel-Sorrentino, R., \& Brissey, C. Concurrent assessment of schedule and intensity control across successive discriminations. Journal of the Experimental Analysis of Behavior, 1975, 23, 247-254.

Rilling, M. E., \& Howard, R. C. The analysis of memory for signals and food in a successive discrimination. In M. L. Commons \& J. A. Nevin (Eds.), Quantitative analyses of behavior: Discriminative properties of reinforcement schedules. Cambridge, Mass: Ballinger, 1981.

Rosen, A. P., \& Terrace, H. S. On the minimal conditions for the development of a peak-shift and inhibitory stimulus control. Journal of the Experimental Analysis of Behavior, 1975, $23,385-414$.

Skinner, B. F. The behavior of organisms. New York: AppletonCentury-Crofts, 1938.

Skinner, B. F. Why I am not a cognitive psychologist. Behaviorism, 1977, 5, 1-4.

Terrace, H. S. Discrimination learning, the peak shift, and behavioral contrast. Journal of the Experimental Analysis of Behavior, 1968, 11, 727-742.

(Manuscript received August 4, 1981; revision accepted for publication April 21, 1982.) 